\title{
THEORY OF OPTICAL TWEEZERS
}

\author{
P. A. Maia Neto and H. M. Nussenzveig \\ Instituto de Física, Universidade Federal do Rio de Janeiro, Caixa \\ Postal 68528, \\ 21945-970 Rio de Janeiro, Rio de Janeiro, Brazil
}

(February 2, 2008)

We derive a partial-wave (Mie) expansion of the axial force exerted on a transparent sphere by a laser beam focused through a high numerical aperture objective. The results hold throughout the range of interest for practical applications. The ray optics limit is shown to follow from the Mie expansion by size averaging. Numerical plots show large deviations from ray optics near the focal region and oscillatory behavior (explained in terms of a simple interferometer picture) of the force as a function of the size parameter. Available experimental data favor the present model over previous ones.

87.80.Cc, 42.50.Vk, 42.25.Fx

Optical tweezers are single-beam laser traps for neutral particles that have a wide range of applications in physics and biology [1]. Dielectric microspheres are trapped and employed as handles in most of the quantitative applications. The gradient trapping force is applied by bringing the laser beam to a diffraction limited focal spot through a large numerical aperture microscope objective.

Typical size parameters $\beta=k a$ ( $a=$ microsphere radius, $k=$ laser wavenumber) range in order of magnitude from values $<1$ to a few times $10^{1}$. A theory of the trapping force based on geometrical optics (GO) [2] should not work in this range. Other proposals (cf. [1]), based on Mie theory, have employed unrealistic near-paraxial models for the transverse laser beam structure near the focus, incompatible with its large angular aperture.

We take for the incident beam before the objective, propagating along the positive $z$ axis, the usual Gaussian $(\mathrm{TEM})_{00}$ transverse laser mode profile, with beam waist $w_{0}$ at the input aperture, where $k w_{0} \gg 1$. We employ the Richards and Wolf [3] representation for the corresponding strongly focused beam beyond the objective, with a large opening angle $\theta_{0}$ (no paraxial assumption), taking due account of the Abbe sine condition. This should be a more realistic representation.

The microsphere, with real refractive index $n_{2}$ (we neglect absorption), is immersed in a homogeneous medium with refractive index $n_{1}$. We consider here the simplest situation, in which the sphere center is aligned with the laser beam axis, so that we evaluate the axial trapping force. With origin at the sphere center, we denote by $\mathbf{r}=-q \hat{\mathbf{z}}$ the focal point position. The fraction $A$ of total beam power that enters the lens aperture is

$$
A=1-\exp \left(-2 \gamma^{2} \sin ^{2} \theta_{0}\right) .
$$

where $\gamma$ is the ratio of the objective focal length to the beam waist $w_{0}$.

By axial symmetry, the trapping force in this situation is independent of input beam polarization: we take circular polarization. The electric field of the strongly focused beam (we omit the time factor $\exp (-i \omega t)$ ) has the Debye-type [3] integral representation

$$
\begin{gathered}
\mathbf{E}_{0}(\mathbf{r})=E_{0} \int_{0}^{2 \pi} d \phi \int_{0}^{\theta_{0}} d \theta \sin \theta \sqrt{\cos \theta} \exp \left(-\gamma^{2} \sin ^{2} \theta\right) \\
\quad \times \exp [i \mathbf{k} \cdot(\mathbf{r}+q \hat{\mathbf{z}})] \hat{\boldsymbol{\epsilon}}(\theta, \phi),
\end{gathered}
$$

where $k=|\mathbf{k}(\theta, \phi)|=n_{1} \omega / c, \hat{\boldsymbol{\epsilon}}(\theta, \phi)=\hat{\mathbf{x}}^{\prime}+i \hat{\mathbf{y}}^{\prime}$, and the unit vectors $\hat{\mathbf{x}}^{\prime}$ and $\hat{\mathbf{y}}^{\prime}$ are obtained from $\hat{\mathbf{x}}$ and $\hat{\mathbf{y}}$, respectively, by rotation with Euler angles $\alpha=\phi, \beta=$ $\theta, \gamma=-\phi$. The factor $\sqrt{\cos \theta}$ arises from the Abbe sine condition.

For each plane wave $\exp (i \mathbf{k} \cdot \mathbf{r})$ in the superposition (2), the corresponding scattered field is given by the well-known Mie partial-wave series [4], in terms of the Mie coefficients $a_{l}, b_{l}$, that are functions of the size parameter $\beta$ and the relative refractive index $n=n_{2} / n_{1}$. By substitution into (2), we obtain the total scattered field $\mathbf{E}_{s}(\mathbf{r})$.

The trapping force is found by replacing the total field $\mathbf{E}=\mathbf{E}_{0}+\mathbf{E}_{s}$ (likewise for $\mathbf{B}$ ) into the Maxwell stress tensor and integrating over the surface of the sphere. The resulting axial force $F$ is proportional to the focused laser beam power $P$,

$$
F=\left(n_{1} / c\right) P Q,
$$

where $Q$ is the (dimensionless) axial trapping efficiency [1].

We denote by $Q_{e}$ the contribution from terms in $E_{0} E_{s}$ and $B_{0} B_{s}$ (that also give rise to the extinction efficiency) and by $Q_{s}$ the remaining terms, so that $Q=Q_{e}+Q_{s}$. We find

$$
\begin{gathered}
Q_{e}=\frac{4 \gamma^{2}}{A} \operatorname{Re} \sum_{l=1}^{\infty}(2 l+1)\left(a_{l}+b_{l}\right) G_{l} G_{l}{ }^{\prime *}, \\
Q_{s}=\frac{-8 \gamma^{2}}{A} \operatorname{Re}\left[\sum_{l=1}^{\infty} \frac{l(l+2)}{l+1}\left(a_{l} a_{l+1}^{*}+b_{l} b_{l+1}^{*}\right) G_{l} G_{l+1}^{*}\right.
\end{gathered}
$$




$$
\left.+\frac{(2 l+1)}{l(l+1)} a_{l} b_{l}^{*} G_{l} G_{l}^{*}\right],
$$

where $G_{l}$ and $G_{l}{ }^{\prime}$ are multipole coefficients for the focused beam,

$$
\begin{aligned}
G_{l}=\int_{0}^{\theta_{0}} d \theta \sin \theta & \sqrt{\cos \theta} \exp \left(-\gamma^{2} \sin ^{2} \theta\right) \exp (i \delta \cos \theta) \\
& \times d_{1,1}^{l}(\theta), \\
G_{l}{ }^{\prime} & =-i \partial G_{l} / \partial \delta,
\end{aligned}
$$

with $\delta=k q$. In (6), $d_{1,1}^{l}(\theta)$ are the matrix elements of finite rotations [5], that can be expressed as

$$
d_{1,1}^{l}(\theta)=\left[p_{l}(\cos \theta)+t_{l}(\cos \theta)\right] /(2 l+1)
$$

in terms of the Mie angular functions [6] $p_{l}$ and $t_{l}$. The results (4) and (5), apart from converging beam effects, have the same structure as the radiation pressure efficiency [6], with which they are closely related, as will be seen below.

In the Rayleigh limit, $\beta \ll 1, Q$ is dominated by the electric dipole Mie term $a_{1}$, and the trapping force (3) becomes $F=(\alpha / 2) \nabla \mathbf{E}^{2}$, where $\alpha$ is the static polarizability of the sphere [1]. In the opposite limit $\beta \gg 1$, the connection with geometrical optics is established by applying to (4) and (5) the following steps [6] [7]. (i) In (6), substitute $p_{l}$ and $t_{l}$ by their (non-uniform) asymptotic expansions for large $l$, and approximate $G_{l}$ and $G_{l}^{\prime}$ by the method of stationary phase 8 . (ii) Compute the average $\langle Q\rangle$ over a size parameter range associated with a quasiperiod of the Mie coefficients. The result is

$$
\begin{aligned}
<Q>_{\mathrm{GO}} & =\frac{4 \gamma^{2}}{A} \int_{0}^{\theta_{0}} d \theta \sin \theta \cos \theta \exp \left(-2 \gamma^{2} \sin ^{2} \theta\right) \\
& \times\left\{\cos \theta+\frac{1}{2} \sum_{j=1}^{2} r_{j} \cos \left(2 \theta_{1}-\theta\right)\right. \\
- & \left.\frac{1}{2} \operatorname{Re} \sum_{j=1}^{2}\left(1-r_{j}\right)^{2} \frac{e^{i\left[2\left(\theta_{1}-\theta_{2}\right)-\theta\right]}}{1+r_{j} e^{-2 i \theta_{2}}}\right\} .
\end{aligned}
$$

In (9), $\theta_{1}=\arcsin (q \sin \theta / a), \theta_{2}=\arcsin \left(\sin \theta_{1} / n\right)$ are the angles of incidence and refraction (defined so as to be negative if $q<0$ ) at the sphere surface associated with a component in the direction $\theta$ of the focused beam (2). The corresponding Fresnel reflectivity for polarization $j$ $\left(\|, \perp\right.$ ) is $r_{j}$. Eq. (9) may also be derived in the framework of GO. Thus, the expression within curly brackets agrees with the GO result for the force exerted by each component ray as first obtained in [9]. The remaining pre-factors in (9), not accounted for previously, represent the intensity distribution of the focused beam as implied by the sine condition and the transverse profile of the laser beam at the input aperture of the objective.

In Fig. $1, Q$ is plotted as a function of $q / a$, the center offset from the focus in units of the sphere radius [10]. The numerical values chosen correspond to the experiment of Ref. [11]: $n_{1}=1.33, n_{2}=1.57, A=0.85, \theta_{0}=$ $78^{\circ}$, which by (1) yield $\gamma^{2}=0.99$. The dotted curve represents the GO result (9). The other curves represent the exact Mie results (4) and (5) for two different $\beta$ values, corresponding to microsphere radii employed in [11]: $1.42 \mu \mathrm{m}$ (dashed) and $2.16 \mu \mathrm{m}$ (solid), respectively $\beta=18.8$ and $\beta=28.4$. The qualitative behavior of the GO curve has been explained [2] in terms of competition between radiation pressure (scattering force) and gradient force. However, the GO result for the maximum backward trapping efficiency $Q_{m}$ is smaller (by a factor of the order of 2) 12] than the values obtained in Ref. [2]. This is in line with the discrepancy between experimental and theoretical values noted in Ref. [13].

The Mie theory provides values for $Q_{m}(0.088$ for $\beta=$ 18.8 and 0.086 for $\beta=28.4$ [14]) below the GO result $Q_{m}=0.095$. The position at which the backward force is maximum lies beyond the corresponding GO value $q / a=$ 1.01. The stiffness decreases as this point is approached from the focus, contradicting the GO prediction and in agreement with an experiment reported in Ref. [13].

GO is also a poor approximation near the geometrical focus, as expected. In fact, Fig. 1 shows that the exact values deviate substantially from GO near $q=0$. The stable equilibrium position shows large positive as well as negative offsets from GO (further discussed below), and the linear Hooke's law range around the equilibrium position is narrower than predicted by GO. Because of the axial focusing effect [6], the (nonuniform) asymptotic approximations to the Mie angular functions employed in the derivation of (9) break down at $q=0$, although (9) is continuous at this point, yielding, with $\theta_{1}=\theta_{2}=0$,

$$
<Q>_{\mathrm{GO}}(q=0)=\frac{4 r}{1+r}<\cos \theta>,
$$

where $r$ is the Fresnel reflectivity for normal incidence and $\langle\cos \theta\rangle$ denotes an average over the intensity distribution of the focused beam (2). Since the incident rays are either backscattered or undeviated in this approximation, (10) represents pure radiation pressure in GO.

The region around $q=0$ deserves special treatment, in view of its relevance to the evaluation of trap axial stiffness. For $\beta \gg 1$, the above discussion and the localization principle imply that the main contributions to (14) and (5) should arise from partial waves with $l \ll \beta$, so that we apply Hankel's asymptotic expansions to the spherical Bessel functions in the Mie coefficients. The results are independent of $l$, and the summations over 
multipole coefficients can then be carried out, resulting in

$$
Q(q=0)=\frac{8 r \sin ^{2} \Delta / 2}{1+r^{2}-2 r \cos \Delta}<\cos \theta>
$$

where $\Delta=4 n_{2} \omega a / c$. This expression corresponds to the radiation pressure efficiency (twice the reflectivity) of an infinite set of parallel-plate interferometers (width $2 a$, refractive index $n_{2}$, so that $\Delta$ is the round-trip phase), each one oriented at an angle $\theta$ with respect to the axis, traversed at normal incidence by the corresponding beam angular component. The GO result (10) follows from (11) by taking an incoherent average. Since $n-1$ is small, we have $r \ll 1$, so that the interferometer reflectivity is nearly sinusoidal.

In Fig. 2, for the same parameters as in Fig. 1, we plot $Q$ at $q=0$ as a function of $\beta$. The Mie curve (full line) displays the expected near-sinusoidal oscillation as $\beta$ increases, approaching the interferometer behavior (11) (shown in dotted line). The GO value (10) (dashed line) is approached in the average sense. The two points corresponding to the $\beta$ values employed in Fig. 1 are shown by circles. Since the radiation pressure at $\beta=28.4$ is above the GO value, the Mie value for the equilibrium position $q_{\text {eq }}$ is larger than the GO result, in agreement with Fig. 1 (the opposite applies at $\beta=18.8$ ). The values for $q_{\mathrm{eq}}$ are found by numerically solving the equation $Q\left(q_{\mathrm{eq}}\right)=0$. In the limit $\beta \gg 1$, they are vanishingly small at $\beta$ values that are minima of $Q(q=0)$. For $\beta \gtrsim 5, q_{\mathrm{eq}} / a$ as a function of $\beta$ oscillates in phase with the oscillations of $Q(q=0)$, around the GO value $\left(q_{\mathrm{eq}} / a\right)_{\mathrm{GO}}=0.217$, and with amplitude of the order of 0.17 .

The trap axial stiffness is given by

$$
\kappa=-\frac{n_{1} P}{c}\left(\frac{\partial Q}{\partial q}\right)_{q=q_{\mathrm{eq}}} .
$$

Within GO, $\kappa$ decreases as $1 / \beta$. This follows from scaling: $Q_{\mathrm{GO}}$ depends on $q$ only through $q / a$. Hence, $\partial Q_{\mathrm{GO}} / \partial q=$ $Q_{\mathrm{GO}}^{\prime}(q / a) / a$, yielding

$$
\kappa_{\mathrm{GO}}=-\frac{n_{1} P}{c} Q_{\mathrm{GO}}^{\prime}\left(\frac{q_{\mathrm{eq}}}{a}\right) \frac{k}{\beta} .
$$

Again for the parameters of Ref. [1] (power $P=3 \mathrm{~mW}$ ), we plot in Fig. 3 the Mie values of $\kappa$ (solid line) [15], the $\mathrm{GO}$ result $\kappa_{\mathrm{GO}}=(18 / \beta)(\mathrm{pN} / \mu \mathrm{m})$ calculated from Eq. (13) (dotted line) and the experimental data points from Ref. [11], with the respective error bars. We also show (dot-dashed line) the values predicted by the electrostatic model recently suggested by Tlusty et al. [16]. As could be expected, their approach may be applied only in the low-frequency (Rayleigh) limit, where it may be replaced by the simpler electric dipole approximation (neglecting the variation of the field over the sphere volume) already discussed above in connection with (画). In order to test the sensitivity of the results to the focused beam parameters, we also plot the Mie values for $\kappa$ corresponding to a larger waist: $\gamma^{2}=0.3$ (dashed line).

For $\beta \gtrsim 10$, the Mie values for $\kappa$ oscillate around the GO curve with period $\Delta \beta=\pi /(2 n)$, like the force $Q(q=0)[$ cf. (11)] and the equilibrium position. This corresponds to a frequency interval $\Delta \nu=c /\left(4 n_{2} a\right)$, which is in the $\mathrm{THz}$ range for spheres with radii of a few microns. As shown in the inset in Fig. 3, where we plot $\left(<\kappa>-\kappa_{\mathrm{GO}}\right) / \kappa_{\mathrm{GO}}$ as function of $\beta$, the average of the Mie values, $\langle\kappa\rangle$, stands above the GO curve, but the relative difference decreases to zero as $\beta$ increases beyond $\beta \approx 55$. For large sphere diameters, $\kappa$ may become very small over short $\beta$ intervals. This may be of interest for applications to scanning force microscopy [11].

In conclusion, by deriving an analytic Mie expansion for the axial trapping efficiency, based on a more realistic model of optical tweezers, we are able to cover the range of interest for most applications. Furthermore, the connection with the correct GO limit (taking into account the sine condition) has been derived from the Mie expansion by size averaging. The behavior near the focus has been obtained and interpreted in terms of an interferometer model, which also accounts for equilibrium position and trap axial stiffness oscillations. These oscillations should be accessible to experiments by scanning the laser beam frequency. Most experimental data points lie above the GO values, closer to the wave optics predictions computed from the Mie expansion employing only the experimentally given parameters.

We thank W. Wiscombe for useful suggestions and programs for quadrature integration and for Mie scattering calculations, and $\mathrm{CNPq}$ for partial support. One of us (P. A. M. N.) acknowledges support by Programa de Núcleos de Excelência (PRONEX), grant 4.1.96.08880.00-7035-1.

[1] A. Ashkin, Proc. Natl. Acad. Sci. USA 94, 4853 (1997), and references therein.

[2] A. Ashkin, Biophys. J. 61, 569 (1992).

[3] B. Richards and E. Wolf, Proc. R. Soc. London A 253, 358 (1959).

[4] C. F. Bohren and D. R. Huffman, Absorption and Scattering of Light by Small Particles (Wiley, New York, 1983).

[5] A. R. Edmonds, Angular Momentum in Quantum Mechanics (Princeton University Press, 1957).

[6] H. M. Nussenzveig, Diffraction Effects in Semiclassical Scattering (Cambridge University Press, 1992).

[7] H. M. Nussenzveig and W. J. Wiscombe, Phys. Rev. Lett. 45, 1490 (1980).

[8] The stationary-phase point ( $q=0$ is excluded) is at 
$\bar{\theta}=\arcsin [(l+1 / 2) / k|q|]$, as expected by the localization principle [6].

[9] G. Roosen, Can. J. Phys. 57, 1260 (1979).

[10] The numerical integrations in Eqs. (6) and (9) were performed with the help of a Kronrod-Patterson adaptative Gaussian-type quadrature method.

[11] M. E. J. Friese et al., Applied Optics 35, 7112 (1996).

[12] The gradient force is overestimated in 22 as a consequence of neglecting the sine condition and the corresponding factor $\cos \theta$ in Eq. (9), which diminishes the contribution of rays at large angles. By the same reason, the stable equilibrium positions as predicted by $(9)$ are further from the focus than the values obtained in [2].

[13] K. Svoboda and S. M. Block, Annu. Rev. Biophys. Biomol. Struct. 23, 247 (1994).

[14] This shows that, in contradiction with the near-paraxial results obtained by Wright et. al. [Appl. Phys. Lett. 63, 715 (1993)], $Q_{m}$ is not a monotonically increasing function of the sphere radius.

[15] The Mie values for $\kappa$ are obtained by deriving from (四) and (5) the partial-wave expansion for $\partial_{q} Q$, and then replacing the results for $q_{\mathrm{eq}}$ into (12).

[16] T. Tlusty et al., Phys. Rev. Lett. 81, 1738 (1998).

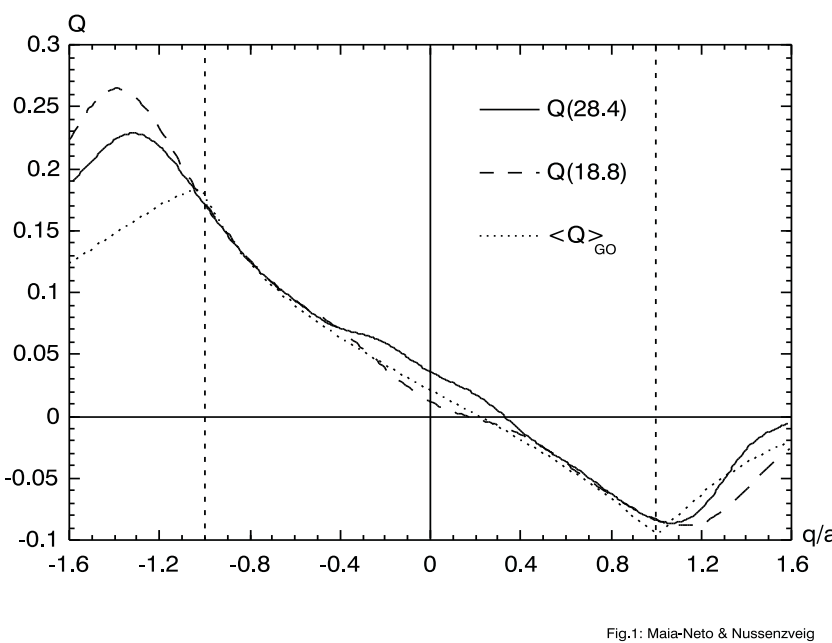

FIG. 1. Normalized axial force versus position (in units of the sphere radius). The dotted line is computed from ray optics theory, whereas the solid and dashed lines are calculated from the wave-optics theory with size parameters $\beta=28.4$ and $\beta=18.8$, respectively. The vertical dashed lines mark the microsphere boundaries.

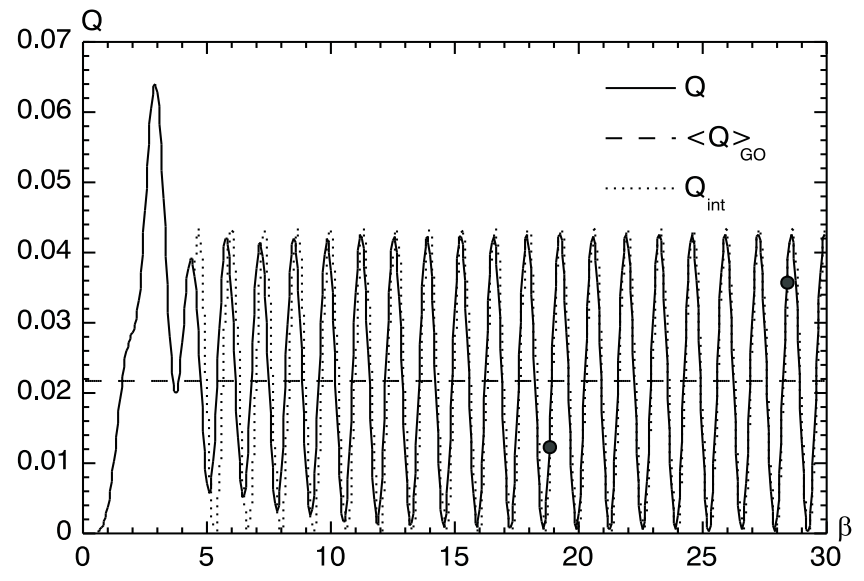

fig. 2: Maia Neto \& Nussenzveig

FIG. 2. Normalized force at the geometrical focal point versus size parameter $\beta$ : exact (full line); interferometer model (dotted line) and geometrical optics (horizontal dashed line). The black circles indicate the two values of $\beta$ used in Fig. 1.

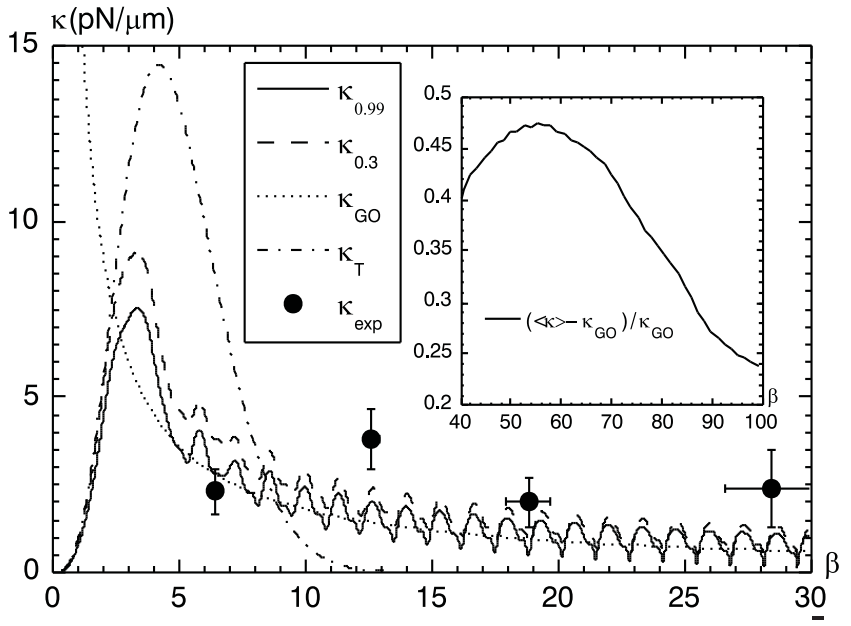

FIG. 3. Axial stiffness $\kappa$ of the optical tweezer as a function of $\beta$. Solid, dotted and dot-dashed lines correspond to the (exact) wave-optics theory, geometrical optics, and electrostatic theory [16], respectively, and for a focal length to waist squared ratio $\gamma^{2}=0.99$. Also shown are the experimental data points of Ref. [11], with corresponding error bars, and the exact values for $\gamma^{2}=0.3$ (dashed line). In the inset, we plot the relative discrepancy between the average of the exact values and the geometrical optics results (for $\gamma^{2}=0.99$ ). 